\title{
Design of plant growth information acquisition and management system based on WIFI
}

\author{
Shaowei Chen, Jing Le, Jian Wang, Ze Wang, Xili Duan \\ Mechanical and Precision Instrument Engineering,Xi'an University of Technology, Xi'an,China
}

\begin{abstract}
A portable plant growth information acquisition and management system based on WIFI was developed to monitor plant growth status by the non - picking leaves. It uses STM32 as main control chip. The chlorophyll characterization value SPAD and water characterization value WI were obtained with the transmittance of plant leaves under the light of 650,940 and $1450 \mathrm{~nm}$, and the fluorescence images were obtained by $460 \mathrm{~nm}$ excitation chlorophyll fluorescence to analyze the infection of the disease. The nitrogen content of the leaves can be retrieved with the fluorescence parameter $R_{f d}$. The software of the upper computer, which adopts the $\mathrm{B} / \mathrm{S}$ architecture, is designed to manage and display the data based on the JAVA language. The stability and reliability of the designed system are verified by field application.
\end{abstract}

\section{Introduction}

Multi-sensor fusion, WSN, Internet of Things and other new technologies make the measurement system more comprehensive, more widely used, and more flexible to form, and they have also been applied in agricultural production and management[1,2]. The measurement systems mentioned above can overcome the shortcomings of single parameter detection to make a comprehensive and detailed judgment of the actual growth of plants. However, the network-based agricultural information monitoring systems currently is mainly to monitor the growth environment of plants[3]. The nutrition that the plant absorbed can not be grasped by them. The non-destructive testes which based on the geometric characteristics of the leaves, fluorescence parameters, etc, mainly detect the single parameter and remain a relatively large error[4]. Therefore, the trend of the comprehensive evaluation of plant growth condition is to carry out long-term tracking measurement of the leaf and multi-parameter detection directly to plant leaves, and the use of plant fluorescence information has the advantage of predicting disease and plant stresses.

A portable monitoring system based on chlorophyll fluorescence and infrared absorption characteristics of crop growth status is developed to achieve the prediction of the main stress in the early crop growth to reach the purpose of accurate irrigation and reasonable fertilization and avoiding the damage to crops. In this paper, the content of nitrogen fertilizer was determined by inducing chlorophyll fluorescence kinetics characteristics, and the distribution of fluorescence images and the change of light intensity were used to predict the degree of disease.
WI and SPAD, which were considered to represent the values of moisture and chlorophyll, were measured by near infrared spectroscopy. To build a data acquisition, preservation, analysis and management platform based on Internet of Things, to provide a non-destructive, in-vivo tracking method for agricultural cultivation, a small multi-functional agricultural information detection system is configured to meet the requirements of the agricultural information age.

\section{Design of measurement system}

The functions of the measurement system include the acquisition, display, storage, processing, remote transmission of data, storage, display of web pages, query and remote control of plant growth signals. STM32F103 is used to manage the acquisition and processing of multiple parameters of plant leaves (all information collection are carried out in the dark room). Considering the factors such as transmission rate and measurement object, this system adopts WIFI as a wireless transmission tool,receive and manages data with PC to achieve long-term monitoring and analysis of plant growth. The system comes with lithium battery power, suitable for field monitoring.Below is a description of each component of the framework shown in Fig. 1.

According to the whole idea of the system, the design scheme is divided into several parts. 


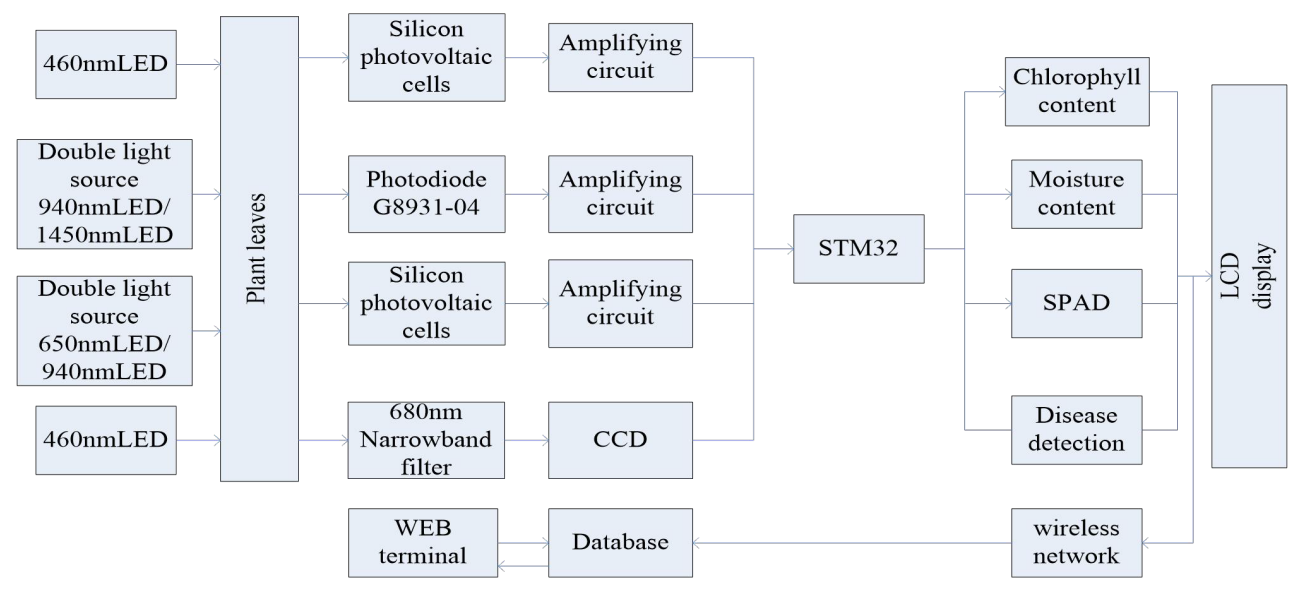

Fig. 1. Overall design block diagram

\subsection{Design of the circuit}

In the acquisition of plants measured signal, the sensor measurement program firstly is determined based on different measured parameters of the spectral characteristics.

\subsubsection{Sensor selection and determination of measurement scheme}

In view of the measurement of plant moisture, it is considered that in the near-infrared absorption region, near-infrared light of $1450 \mathrm{~nm}$ is easily transmitted through the plant leaves, the near-infrared light of $980 \mathrm{~nm}$ is not, and to reduce the thickness of the blade and the influence of transmission process irregular optical path on measurement results as far as possible. Therefore, in this measurement system, we select the high-power LED with a wavelength of $1450 \mathrm{~nm}$ as the measuring light source, and select the $980 \mathrm{~nm}$ high-power LED as the contrast light source.

In the photoelectric sensor, the photo-multiplier tube has poor noise resistance and high power requirements. This creates an obstacle at field measurements, and also the response frequency of the photocell can not meet these experiment requirements. Therefore, Hamamatsu G8931-04 indium gallium arsenide photo-diode is selected, which wavelength range detected is $800-1700 \mathrm{~nm}$.

Plant nitrogen fertilizer measurements can take advantage of a feature that nitrogen fertilizer content and chlorophyll content have a definite relationship. Plant nitrogen content can be inversion by experiment, chlorophyll content measurement can be used in the following two ways.

Chlorophyll is more sensitive to blue light $(400-500 \mathrm{~nm})$ and red $(600-700 \mathrm{~nm})$. Therefore, the principle of SPAD detection is selecting the red light area as the main band for measuring chlorophyll according to the absorption rate of chlorophyll spectrum. Similar to the measurement of moisture, to eliminate the thickness of the blade and other aspects of the measurement results in infrared light emission and reception, the $650 \mathrm{~nm}, 940 \mathrm{~nm}$ light-emitting diodes are selected as the excitation light source, the silicon photo-voltaic diode 2DU10 is used to detect.

The system can also obtain the chlorophyll content by using $460 \mathrm{~nm}$ to induce chlorophyll fluorescence, then through a narrow band filter with a wavelength of $680 \mathrm{~nm}$ and finally receiving fluorescence signal by the silicon photo-voltaic diode 2DU10.

The plant disease detection and early prediction can be achieved by using a CCD camera to capture the fluorescence images induced by $460 \mathrm{~nm}$ LED.

After determining the measurement scheme and the sensor, it is necessary to carry out the circuit design and conditioning.

\subsubsection{Signal process module}

According to the design requirements, the signal acquisition circuit needs $\pm 12 \mathrm{~V}$ power supply. Selecting two $12 \mathrm{~V}$ portable rechargeable batteries, the protection circuit designed to meet the system requirements and to avoid the damage that hardware circuit components suffered under the over-voltage, over-current conditions and other circumstances.

Analog signal conditioning circuit module is mainly to adjust the photoelectric signal meeting the acquisition voltage range of $\mathrm{ADC}$, it is convenient for subsequent analysis and processing. The conditioning circuit mainly includes the $\mathrm{I} / \mathrm{V}$ conversion circuit and the amplifier filter circuit.

As shown in Fig.2, AD795 is used as the preamplifier (maximum input bias current is $1 \mathrm{pA}$ and the maximum offset voltage is $250 \mu \mathrm{V}$ ), and the second stage amplifier is AD620. The output signal is within $0-3.3 \mathrm{~V}$.

In order to use conveniently, ensuring the minimal impact of interference factors such as the outside light, the location of the leaves placed and so on, several independent photoelectric conversion probes are designed by ourselves that photoelectric conversion and follow-up circuit are installed into an organic whole. 


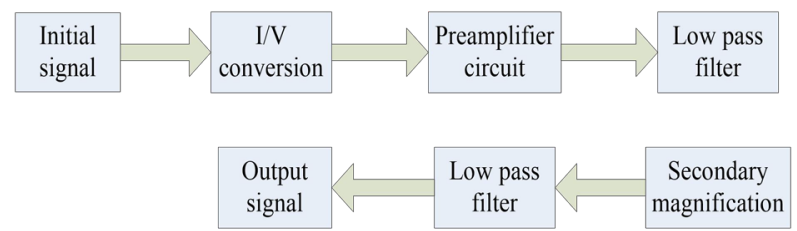

Fig. 2. Signal conditioning flow chart.

\subsection{MCU module}

Acquisition and control is achieved by STM32F103, which controls the acquisition and storage of plant growth information by pressing the key to control internal $\mathrm{ADC}$, then the corresponding characterization parameters are calculated. Indicators are used to indicate the operation of the program, while the AT command is used to transfer data between MCU and the monitoring center.

For the acquisition of moisture in the leaves, it is necessary to alternately irradiate the photoelectric receivers with two states of no-load and placed leaves, then the water characterization value WI according to Bill-Lambert's law[5] is obtained by equation (1).

$$
W I=K \lg \left\{\frac{U_{2} / U_{1}}{U_{4} / U_{3}}\right\}
$$

( $K$ is a constant, $U_{1}$ is the voltage corresponding to the light intensity of $980 \mathrm{~nm}$ passing through the blade, $U_{2}$ is the voltage corresponding to the light intensity at no load; $U_{3}$ is the voltage corresponding to the light intensity of $1450 \mathrm{~nm}$ passing through the blade, $U_{4}$ is the voltage corresponding to the light intensity at no load. This process needs to collect two channel signals.)

Similarly, the photoelectric receivers were alternately irradiated with no-load and placed leaves to obtain the chlorophyll characterization values SPAD:

$$
S P A D=K \lg \left\{\frac{I R_{t} / I R_{0}}{R_{t} / R_{0}}\right\}
$$

( $K$ is a constant, $I R_{\mathrm{t}}$ is the light intensity of $940 \mathrm{~nm}$ passing through the blade, $I R_{0}$ is the intensity of the emitted light, $R_{t}$ is the received light intensity of $650 \mathrm{~nm}$ passing through the blade, while $R_{0}$ is the intensity of the emitted red light. This process needs to collect two channel signals.)

In addition, the chlorophyll fluorescence induced by $460 \mathrm{~nm}$ was used to collect the characteristic points of the fluorescence kinetics curve after 15 min dark adaptation. The fluorescence characteristic parameter $R_{f d}$ was calculated to invert the nitrogen content of the leaves.

$$
R_{f d}=\left(F_{m}-F_{s}\right) / F_{s}
$$

( $F_{m}$ is the maximum fluorescence value, $F_{s}$ is the steady-state fluorescence yield, measured in the saturated pulse light $3 \min \sim 4 \min [6]$.)

So the MCU need to acquire the five channel signals with internal ADC. Below is a description of Signal acquisition and processing process shown in Fig. 3.

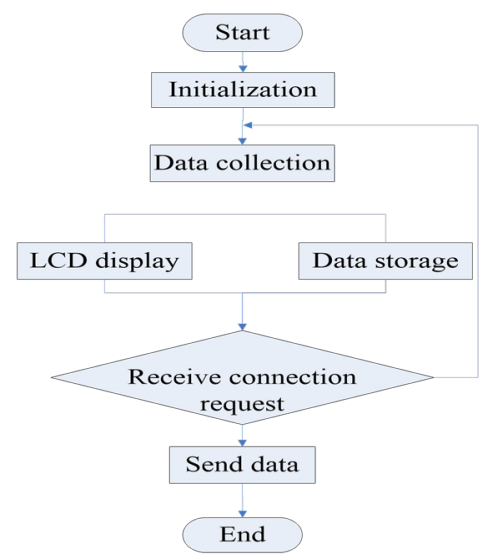

Fig. 3. Signal acquisition and processing process.

\subsection{Wireless network coverage and access}

The wireless network acts as a bridge between the nodes and the monitoring stations, which plays an important role in the whole measurement system. As a server, low-power ESP8266 is configured as AP, TCP / UDP Client transparent mode to achieve data transmission. When the module does not receive data, it will be moved into deep-sleep state. Its power consumption will be as low as $10 \mu \mathrm{A}$.

After the MCU completes the data acquisition and processing, we can send the processed leaf water, fertilizer and chlorophyll information to the monitoring terminal through the AT command and generate the spreadsheet to facilitate the import of the database. The fluorescence images captured by the CCD are transmitted to the monitoring center through the map transmission module, and the images will be processed to obtain the $R_{f d}$ distribution of each pixel point to observe the change of the disease area.

\subsection{Information monitoring and management}

\subsubsection{The overall design of the monitoring center}

For the monitoring and management part, we construct a plant information transmission and management system based on JAVA language. MySql technology was used to establish a database to store the transported data, JSP technology was used as a tool for building the system terminal page, and a connection between website and database was established by JDBC technology. The Web includes users management (registration, logout, logout, etc.), data management (plant location, time, information), and reference information (weather, agricultural advice, etc.), users can use the website to search for plant growth information.

As shown in Fig. 4, the farmland information management system is divided into three layers: view layer, control layer and model layer. Then the model layer is divided into two layers: one layer is the service layer, the other is the data access layer. The data access layer encapsulates the most basic database connection methods provided by JDBC. The service layer 
encapsulates the data access layer, which can improve the system's scalability.

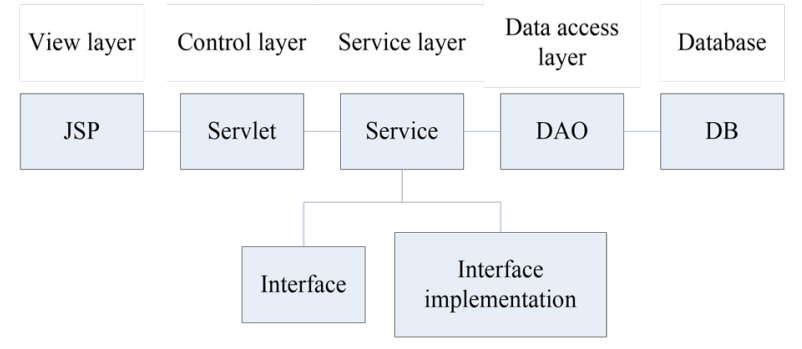

Fig. 4. System structure of information management

\subsubsection{Interface and function design}

The Login interface function is achieved by JSP technology. After the user enters the account number and password, the control layer Servle will obtain and verify the user information, the verification method provided by the model layer. After successful landing, users can enter the main interface. The management system includes four function modules: personnel management, crop data management, farmland data management and MCU management. Users can jump through the corresponding function interface by clicking these modules.

1) Personnel management: Users can add, delete, modify and query the staff;

2) MCU management: Controlling MCU uploads crop information, and import information into the database through the JAVA Socket class;

3) Crop data management: Users can query the corresponding crop of water, nitrogen, chlorophyll, disease information, and the information can be displayed through the form of digital curve, and imported into the spreadsheet;

4) Farmland data management: The different farmland can be increased, deleted, modified and queried. The information display is implemented by JSP hyperlink tags and JavaScript, which mainly provides some agricultural knowledge and real-time agricultural information. The software interface is shown in Fig. 5.

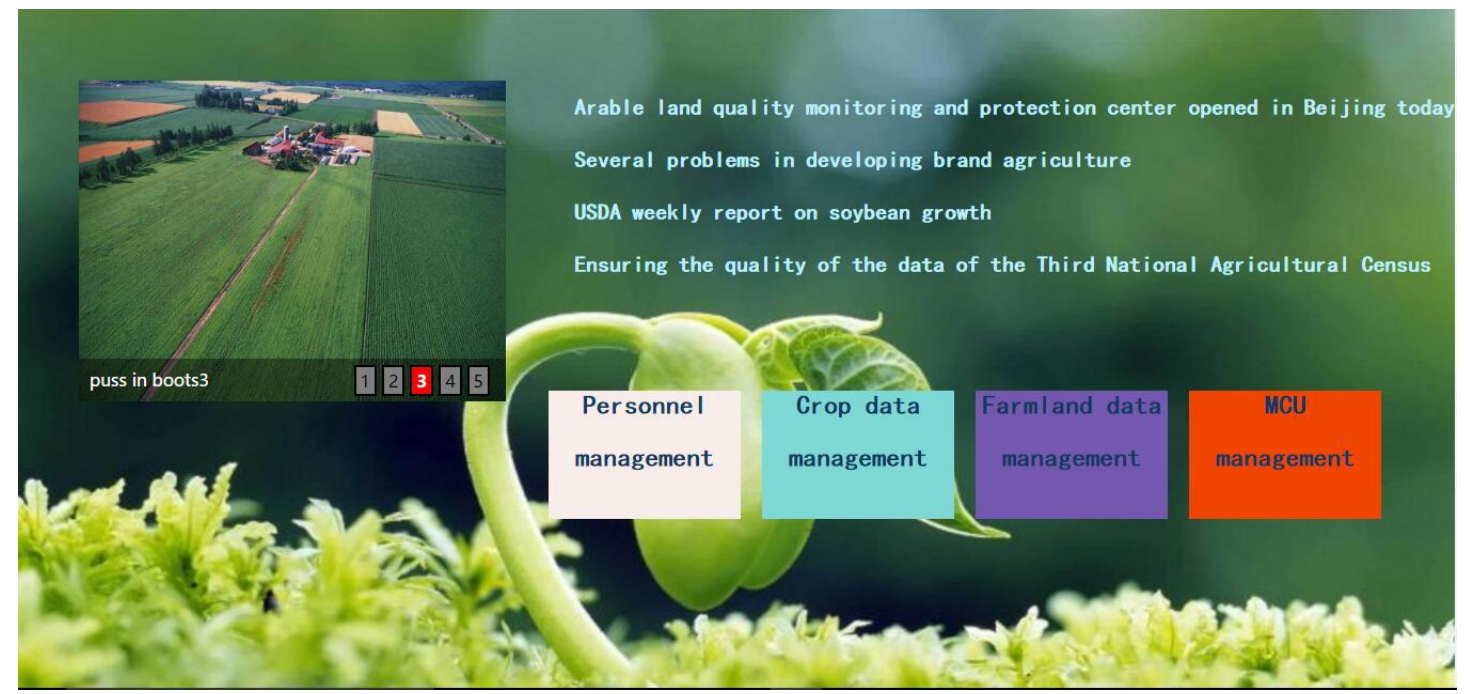

Fig. 5. Home page display.

\section{System testing and analysis}

The system can observe the growth status of plants under different conditions, such as water stress, fertilizer stress, early prediction of disease, etc. There are some experimental data to clarify the system's practicality and feasibility.

56 slices of green radish and 30 pieces of heather were selected in the experiment. The values of WI and SPAD and fluorescence intensity of the selected samples were measured by the designed system. The chlorophyll concentration and water content were obtained by spectrophotometry and drying method. It can be seen from Fig. 6 that the moisture content is in direct proportion to the value of WI, and the relative error of the mid-waist part of the plant leaves is relatively small, which can best reflect the real moisture content of the plant leaf. The relationship between chlorophyll content and the value of SPAD is similar to the quadratic polynomial model, what is the same as nitrogen fertilizer content and $R_{f d \text {. }}$
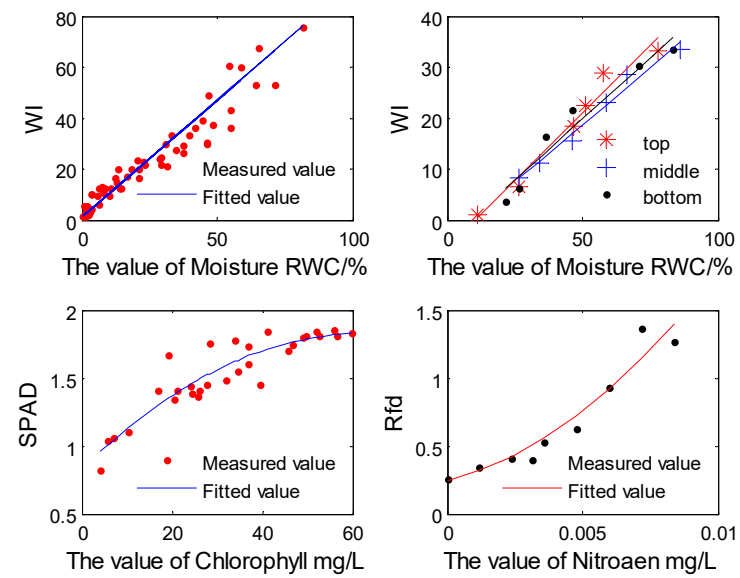

Fig. 6. The fitting curve of WI,SPAD and $R_{f d}$

The $R_{f d}$ diagram of leaves was obtained by using $\mathrm{C}++$ 
to analyze the fluorescence images of green radish and rose leaves. The relationship between the mean value of $R_{f d}$ and the degree of infection was analyzed and the pseudo color images of green leaves[7] were shown in Fig. 7,8.
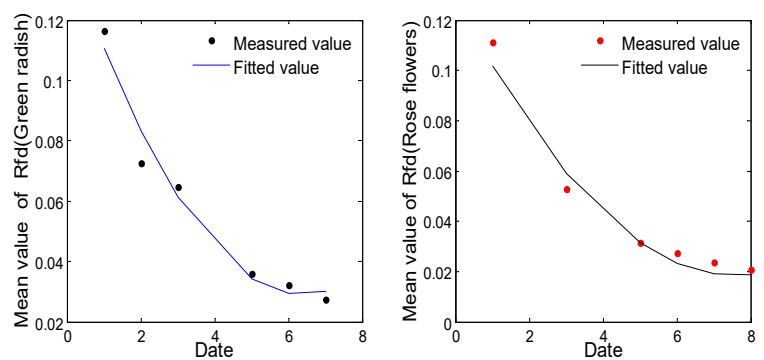

Fig. 7. Curve of infection degree and $R_{f d}$
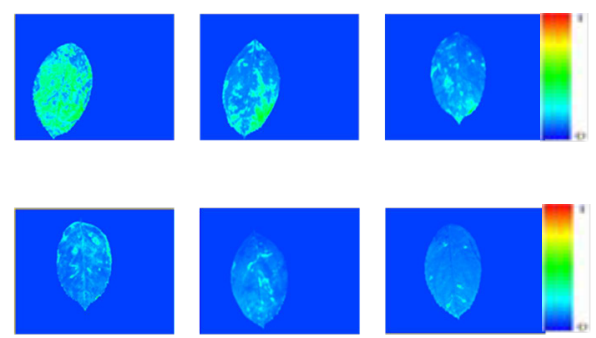

Fig. 8. Pseudo-color map of green radish leaves with increased degree of infection

The relationship between the degree of infection and $R_{f d}$ value of green radish and rose is more consistent with quadratic polynomial model according to Fig. 7. With the increase of the degree of infection, the fluorescence characteristic parameter $R_{f d}$ will be reduced to a certain stationary value.

The results of different experiments show that the measured value can reflect the real growth information of crops well. Therefore, the system designed in this paper has good reliability and can be used for inversion of crop water, fertilizer and chlorophyll.

\section{Concluding}

In this paper, the WIFI-based agricultural information acquisition and management system designed for crop growth problem is portable, a wide covering range of structure, simple and stable. This paper mainly presents a detailed design scheme including constructs of the hardware and software system, the measurement principle completed the experimental verification. This system can achieve the database establishment, invert the chlorophyll, water and nitrogen fertilizer content of the crop, and analyze the disease and the stress. Meanwhile using these parameters, the influence of the crop growth state can be analyzed by the multi-sensor information fusion technology. The research can provide some technical support to achieve the agricultural input-output optimization and the economic benefit maximization.

This work has been supported by the special project of Agricultural Machinery Administration of Shaanxi Province (Shaanxi Agricultural Machinery number [2017]4). Sincere thanks for the comments and contributions of anonymous reviewers and members of the editorial team.

\section{References}

1. Yong H, Pengcheng N, Fei L, Transactions of the Chinese Society for Agricultural Machinery, 44, 10, 216-226, (2013).

2. Sokratis Barmpounakis, Alexandros Kaloxylos, Aggelos Groumas, et al, INFORMATION PROCESSING IN AGRICULTURE, 2, 51-63, (2015).

3. Suryakant Sawant, Surya S. Durbha, Jagarlapudi Adinarayana, Computers and Electronics in Agriculture, 138, 175-187, (2017).

4. Wenbo W, Dengxin H, Jing L, Meixia L, Ning C, Acta Physica Sinica, 62, 19, 190601-1-6, (2013).

5. Chunyan Z, Dengxin H, Jing L,Spectroscopy and Spectral Analysis, 35, 9, 2629-2633, (2015).

6. Claus Buschmann,Hartmut K.Lichtenthaler, Plant Physiology, 152, 297-314, (1998).

7. Haibo L, Jing S, Zhenxun Y, Detailed explanation of digital image processing technology of Visual $\mathrm{C}++$ (Machinery Industry Press, 2014). 